\title{
RDUS
}

Revue de DROIT

UNIVERSITÉ DE SHERBROOKE

Titre : $\quad$ LA PROTECTION DE L'EAU EN VERTU DE L'ARTICLE 982 C.C.Q. :

PROBLÈMES D'INTERPRÉTATION

Auteur(s): $\quad$ Charlotte LEMIEUX

Revue : $\quad$ RDUS, 1992-1993, volume 23, numéro 1

Pages: $\quad 191-200$

ISSN : $\quad 0317-9656$

Éditeur : $\quad$ Université de Sherbrooke. Faculté de droit.

URI : $\quad$ http://hdl.handle.net/11143/13385

DOI : https://doi.org/10.17118/11143/13385 
Page vide laissée intentionnellement. 


\section{LA PROTECTION DE L'EAU EN VERTU DE L'ARTICLE 982 C.C.Q. : PROBLÈMES D'INTERPRÉTATION}

par Charlotte LEMIEUX*

SOMMAIRE

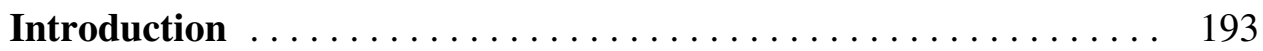

Le filtre de l'intérêt général . . . . . . . . . . . . . . . . . 194

Le rôle des tribunaux ...................... 195

Le droit à l'usage de l'eau $\ldots \ldots \ldots \ldots \ldots \ldots \ldots \ldots \ldots$

Le statut de l'eau ......................... 198

Les titulaires du recours et l'intérêt requis . . . . . . . . . . 199

Les personnes et les actes visés par le recours .......... 200

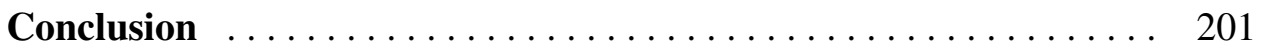

*. Professeure à la Faculté de droit de l'Université de Sherbrooke. 

l'article 982 C.c.Q. : problèmes d'interprétation

(1992) 23 R.D.U.S. 


\section{Introduction}

Sans doute en raison de contraintes nouvelles, la tendance moderne est à l'effacement des droits individuels devant les impératifs collectifs. Le droit de propriété fait partie de ces droits dont la force s'estompe au fil des ans, et des lois particulières ${ }^{1}$. En France, c'est vers le début de ce siècle, mais toujours au nom de l'intérêt général, qu'ont commencé à poindre les premières interventions du législateur restreignant le droit de propriété ${ }^{2}$. Au Québec, la Loi sur la protection du territoire agricole ${ }^{3}$, la Loi sur l'aménagement et l'urbanisme ${ }^{4}$, et la Loi sur la qualité de l'environnement ${ }^{5}$ ont soumis les propriétaires fonciers à l'emprise de l'intérêt collectif. Plus ancien, mais partiellement jugulé par une application assez rigoureuse, l'article 407 C.c.B.C avait fait de même, permettant l'expropriation pour cause d'utilité publique. Quant aux limites légales imposées par le Code civil du Bas Canada, que le nouveau code range maintenant parmi les «règles particulières à la propriété immobilière» ${ }^{6}$, elles participaient d'un autre esprit : fondées sur un intérêt individuel concret, immédiat, et circonscrit, elles se trouvaient atténuées, et justifiées, par leur réciprocité. Mais on peut affirmer que, jusqu'ici, c'est au nom de l'intérêt général et par des lois particulières que le droit à la libre disposition des biens a, la plupart du temps, été entamé. Et lorsque ce droit a été entamé en faveur d'intérêts particuliers, il s'agissait de restrictions symétriques, dont tous souffraient, au profit de chacun.

Dans le contexte de dirigisme économique et de protectionnisme social qui est devenu le nôtre, l'article 982 du Code civil du Québec pourrait sembler le bienvenu. Cet article crée un nouveau recours en faveur de tous ceux qui ont «droit à l'usage d'une source, d'un lac, d'une nappe d'eau ou d'une rivière souterraine, ou d'une eau courante». Ces derniers pourront désormais exiger la

1. La transformation du droit de propriété a été imputée à l'ingérence du droit public dans le domaine de la propriété privée (Michel POURCELET, «L'évolution du droit de propriété depuis 1866», dans Le droit dans la vie économico-sociale, Montréal, P.U.M., 1970, p. 3, à la p. 10).

2. $\quad$ A.-M. PATAULT, Introduction historique au droit des biens, Paris, P.U.F., 1989, p. 269.

3. $\quad$ L.R.Q., c. P-41.1.

4. $\quad$ L.R.Q., c. A-19.1.

5. L.R.Q. c. Q-2.

6. Articles 976 et suiv. 
destruction ou la modification de tout ouvrage qui pollue ou épuise l'eau dont ils ont l'usage, ou peut-être même qui menace de le faire, puisque le recours cherche à «éviter» la pollution ou l'épuisement de l'eau. Cette innovation de l'article 982 C.c.Q., qui semble s'inscrire adroitement dans le discours actuel où l'environnement prédomine, est-elle condamnée à rester lettre morte, ou est-elle au contraire susceptible de créer des bouleversements mal venus et des remous inattendus?

Nous nous proposons, après avoir examiné la limite de l'intérêt général, de souligner le rôle accru qui sera dévolu aux tribunaux, et les problèmes que ces derniers pourraient avoir à résoudre. Parmi ces problèmes, l'identification des bénéficiaires du recours vient en première place. Après quoi nous serons en mesure d'en comprendre l'ampleur virtuelle, ampleur qui pourrait être jugulée, ou du moins atténuée, par une interprétation plus conforme à l'esprit du code qu'à la lettre de son article 982.

\section{Le filtre de l'intérêt général}

L'article 982 n'étonne pas en ce qu'il contribue à l'érosion progressive du droit fondamental qu'est le droit de propriété, mais il étonne par les motifs au moyen desquels il justifie cette érosion. En effet, la restriction de l'article 982 s'applique à moins d'aller à l'encontre de l'intérêt général. Il ne s'agit donc pas d'une restriction imposée à tous dans l'intérêt de chacun, ce qui lui donnerait soit le caractère réciproque, soit la rentabilité sociale des actuelles limites du droit de propriété. Il s'agit plutôt d'une restriction imposée à certains propriétaires, dont les constructions et ouvrages risquent de polluer l'eau éventuellement, mais pas à d'autres, dont les constructions et ouvrages la polluent effectivement, et pourront continuer à la polluer ou à l'épuiser dans l'intérêt général.

Cet intérêt général permettant d'échapper à la restriction de l'article 982 C.c.Q. reste à définir, mais comme il s'agit d'une notion floue, élastique, variable, mobile, et soumise au contexte social, économique et culturel, sa définition fera problème. À titre d'exemple, on peut déjà prévoir que de nombreuses entreprises pourraient tenter de faire valoir que leurs constructions polluantes offrent un intérêt économique général. Le critère de l'intérêt général pourra ainsi, suivant l'interprétation qu'en feront les tribunaux, rendre le recours inefficace pour obtenir la modification de barrages ayant un impact désastreux sur la quantité et la qualité de l'eau, mais par contre efficace pour obtenir la démolition du quai d'un voisin riverain.

Par ailleurs, la généralité de l'article laisse prévoir que les groupes pour la défense de l'environnement chercheront à l'invoquer pour obtenir la destruction d'innombrables ouvrages. La conjugaison des articles 913, 920 et 982 du Code pourrait également, dans une interprétation libérale, aboutir à la 
destruction de constructions à la demande de personnes n'ayant pas, autrement, l'intérêt requis en vertu de l'article 55 C.p.c.

\section{Le rôle des tribunaux}

Le sort du nouveau recours de l'article 982 C.c.Q. reposera donc entièrement sur l'interprétation qu'en feront les tribunaux. Â travers la définition de l'intérêt général, à travers l'emploi du mot éviter, et à travers l'intérêt requis, on peut prévoir que la portée du recours sera vivement questionnée. Si les tribunaux tendent à donner au recours une portée étendue, et s'ils exigent de surcroît un intérêt minimal à poursuivre, le droit de propriété de tous les riverains s'en trouvera démesurément affaibli. Mais si, d'autre part, ils interprètent largement la notion d'intérêt général, l'article 982 C.c.Q. ajoutera peu aux dispositions qui le précèdent.

On peut regretter que le Législateur confie en quelque sorte une partie de la gestion de l'eau au pouvoir judiciaire, et, sans parler d'abdication de sa part, on peut s'étonner de ce transfert qui menace l'étanchéité des pouvoirs, en imposant au judiciaire un fardeau qui ne lui revient pas nécessairement. En effet, la gestion de l'eau relève actuellement de comités administratifs, mais de plus en plus les tribunaux de droit commun seront appelés à déterminer ce qui pollue ou épuise l'eau, ce qui est susceptible de la polluer ou de l'épuiser, et ce qui pourrait en éviter la pollution ou l'épuisement. Il se pourrait même que le recours cache, sous ses allures de recours en faveur de l'usager, un recours en faveur de ce qu'il est désormais convenu d'appeler l'environnement. De la même façon que l'article 980 al. 2 C.c.Q. crée une obligation qui, ne pouvant favoriser aucun usager plausible ${ }^{7}$, a vraisemblablement été établie en faveur de l'eau ellemême.

Pourtant le droit civil est par tradition fondamentalement anthropocentrique. Qu'il traite des liens entre les personnes, ou entre les personnes et les biens, c'est toujours du point de vue de la personne qu'il le fait. Or le recours de l'article 982 ne vient pas sanctionner un lien de droit entre l'eau et son usager potentiel. À travers le filtre de l'intérêt général et le fantôme d'un utilisateur aléatoire, il vient surtout consacrer l'importance de préserver l'environnement, s'inscrivant ainsi dans une mode qui devrait rester étrangère au Code.

7. Soit que personne ne puisse invoquer un manquement à l'obligation prévue à l'article 980 al. 2, qui est une obligation pour le propriétaire de conserver la qualité de l'eau d'un lac entièrement situé sur son fonds, soit que toute personne puisse le faire, et dans ce dernier cas, la même possibilité existerait logiquement en ce qui concerne l'article 982. 
Mais que l'article 982 C.c.Q. vise à protéger le droit d'usage de l'eau et ses titulaires, ou qu'il vise à protéger l'eau elle-même, ce sont deux hypothèses également critiquables, car dans le premier cas, le «droit à l'usage» de l'eau est placé dans une position hiérarchiquement supérieure au droit de propriété, ce qui est injustifiable, et dans le second cas, une dimension étrangère à la nature même du droit civil se trouve introduite dans une codification, dont on n'attend pas qu'elle vienne régir les rapports entre le citoyen et l'environnement.

\section{Le droit à l'usage de l'eau}

Le droit «à l'usage d'une source, d'un lac, d'une nappe d'eau ou d'une rivière souterraine, ou d'une eau courante...» n'est vraisemblablement que le droit à l'usage de l'eau elle-même, puisque la seule façon d'utiliser une nappe d'eau ou une rivière souterraine est d'utiliser leur eau, et que la seule façon d'utiliser une source, ce terme pouvant désigner soit l'eau elle-même, soit l'issue par laquelle elle jaillit du sol, est également d'en utiliser l'eau. Quant au droit d'usage d'un lac, il ne peut aller au-delà du droit à l'usage de l'eau qu'il contient, puisqu'il n'y a pas non plus d'autre façon d'utiliser un lac qu'en en utilisant soit son lit, soit son eau, et que son lit fait l'objet d'un droit de propriété (en vertu des deux alinéas de l'article 919 C.c.Q.) dont aucune disposition ne vient limiter l'exclusivité. Les titulaires du droit à «l'usage d'une source, d'un lac ou d'une eau courante» n'ont, en fait, qu'un droit à l'usage de l'eau.

Mais ce droit d'usage qu'exige l'article 982 pour son application n'est pas le même droit d'usage qui «constitue une prérogative distincte et (...) primordiale de la propriété» ${ }^{8}$. Puisque l'eau ne peut faire l'objet d'aucune appropriation privée (sinon partiellement, par mise en récipient) le «droit d'usage» qu'exige le recours de 982 C.c.Q. est un droit complètement étranger au droit de propriété, et n'en constitue pas l'un des trois attributs (usus, fructus, et abusus). Cet usage dont il est question est la simple faculté d'utiliser une eau qui n'appartient à personne, faculté que le code érige, peut-être par inadvertance, en droit d'usage. Et on ne pourrait efficacement objecter que l'eau appartient à tous et chacun, puisque ce n'est pas le propre des choses communes de faire l'objet d'un droit de propriété, même collectif.

De sorte que, jusqu'à preuve du contraire, et à moins que le nouveau recours ne crée par ricochet le nouveau principe d'un droit de propriété communautaire $^{9}$, l'usage identifié par l'article 982 n'est rien de plus que la possibilité d'utiliser une chose qui est à la disposition de tous, tout en

8. $\quad$ Gérard CORNU, Droit civil, $5^{\mathrm{e}}$ éd., Paris, Montchrestien, 1991, n 1026 , p. 335.

9. Mais toute sanction découle d'un droit, et non l'inverse. La Common Law procède autrement toutefois, postulant qu'il n'existe aucun droit là où il n'y pas de sanction. 
n'appartenant à personne. Il est étonnant qu'au nom d'un droit aussi faible dans la hiérarchie du droit des biens et de la propriété, le Code ait choisi de créer un recours limitant le droit des propriétaires de se doter d'ouvrages et de constructions susceptibles de polluer ou d'épuiser l'eau. Il est vrai que ce recours, spectaculaire de prime abord, pourrait être édenté par une interprétation large de ce qu'est l'intérêt général, et par l'exigence d'un intérêt distinct à l'usage de l'eau.

\section{Le statut de l'eau}

La res communis est «une chose n'appartenant à personne, mais dont tout le monde a l'usage» ${ }^{10}$. Elle est à la disposition de chacun, tout en n'appartenant à personne. Il ne faut pas la confondre avec la chose, et encore moins avec le bien, destiné à l'utilité publique. Ce n'est pas par sa destination qu'une chose commune se qualifie, mais par le fait qu'elle n'est pas susceptible d'appropriation. Le Code lui-même confirme cette distinction, par ailleurs connue, de la façon suivante : l'article 913 al.1 C.c.Q. pose le principe que les choses communes ne sont pas susceptibles d'appropriation, mais l'alinéa deuxième énonce ensuite l'exception de deux choses communes, l'air et l'eau, lesquels pourront faire l'objet d'une appropriation partielle, mais à la condition de ne pas être destinées à l'utilité publique. C'est donc que les choses communes peuvent être ou ne pas être destinées à l'utilité publique.

Le seul fait que tous puissent circuler sur les cours d'eau, suivant l'article 920 C.c.Q., ne peut suffire à destiner leur eau à l'utilité publique, auquel cas cette eau ne serait jamais susceptible d'appropriation par mise en récipient, car l'article 913 al. 2 du Code ne permet que l'appropriation de l'eau non destinée à l'utilité publique. Ce deuxième alinéa de l'article 913 ne peut recevoir d'effet que si l'usage commun (ou usage public) et l'utilité publique sont deux notions distinctes.

En conséquence, qu'elle soit ou non destinée à l'utilité publique, l'eau appartient à cette catégorie des choses communes. L'article 913 C.c.Q., dont le premier alinéa empêche l'appropriation des choses d'usage commun, et dont le deuxième alinéa crée une exception à propos de l'eau, montre que l'eau est bel et bien visée par la règle du premier alinéa, et constitue une chose commune. L'article 920 C.c.Q., qui permet à tous de circuler sur les cours d'eau et les lacs, en respectant certaines conditions, va dans le même sens. L'eau étant une chose commune, tous en ont l'usage. Se pourrait-il que tous les usagers potentiels de l'eau puissent invoquer l'article 982 C.c.Q.? 


\section{Les titulaires du recours et l'intérêt requis}

À notre avis, l'article 982 C.c.Q. ne peut être réduit à un simple complément des articles 981 et 980 . Nous ne croyons pas non plus qu'il faille le voir comme étant disponible seulement aux personnes dont le rapport avec l'eau est établi à l'article 981 C.c.Q., puisqu'il a la particularité d'énumérer toutes les sortes d'eau courante ou dormante qui existent, ce qui n'est pas le cas des articles précédents. Quoique d'application plus large, le recours de 982 pourrait sans doute être utilisé comme sanction des obligations créées à 980 et 981 C.c.Q.

La conséquence de son autonomie et de sa généralité pourrait être de le rendre disponible à toute personne ayant droit à l'usage de l'eau sous toutes ses formes descriptives, ce qui pose la question de l'intérêt à poursuivre. Sans doute faut-il raisonnablement s'attendre à ce qu'elle soit soulevée, en particulier dans des causes-types portées devant les tribunaux par les groupes de protection de l'environnement, qui se verront opposer leur intérêt insuffisant et indistinct. Ces derniers pourraient faire valoir que toute personne a l'intérêt requis en vertu de l'article 55 C.p.c. et en satisfait les conditions, puisque chacun a droit à l'usage de l'eau, ou encore que l'article 982 lui-même accorde l'intérêt requis, du seul fait qu'il est ouvert à «celui qui a droit à l'usage de l'eau». Dans la même veine, l'article 980 al. 2 pourrait leur servir à démontrer que toute personne a un intérêt suffisant à poursuivre lorsque la qualité de l'eau est menacée, à défaut de quoi il s'agirait d'un article inapplicable, puisqu'il créerait alors une obligation dépourvue de bénéficiaire. Plus généralement, ils pourront plaider que tous ont un intérêt suffisant à la qualité de l'eau, puisque chacun a droit à la qualité de son environnement, ceci en vertu de l'article 19 de la Loi sur la qualité de l'environnement $^{11}$. Il est à prévoir que de tels litiges deviendront chose fréquente dès l'entrée en vigueur du nouveau code.

Si le seul droit à l'usage de l'eau devait conférer l'intérêt requis, l'article 982 C.c.Q. constituerait à coup sûr une révolution apparente. Une révolution, car reconnaître l'intérêt requis à des demandeurs n'exerçant pas effectivement leur droit d'usage de l'eau, et pouvant même être dans l'impossibilité de l'exercer $^{12}$, donnerait à chacun un droit de regard sur les constructions d'autrui. Mais une révolution apparente, car la limite de l'intérêt général empêcherait le recours innovateur de s'appliquer aux ouvrages et constructions jugées d'intérêt général. Plusieurs sociétés polluantes, et parmi les plus grandes, échapperaient

11. Précitée, note 5. Une interprétation assez restrictive de cet article a toutefois prévalu jusqu'ici.

12. Il peut être difficile par exemple d'utiliser l'eau d'un lac dont le lit est privé, sans prendre pied sur ses berges. 
ainsi à la surveillance de l'article 982. C'est parmi les plus grandes sociétés que se retrouvent celles qui, premièrement, risquent davantage de diminuer la qualité ou la quantité de l'eau et qui, deuxièmement, sont susceptibles d'être jugées d'intérêt général. Bref, si tous peuvent agir, le recours est aussi révolutionnaire qu'excessif, mais s'ils ne peuvent agir que contre de petits pollueurs (ou des voisins détestés), le recours perd de son envergure, tout en gardant son aspect politiquement correct.

\section{Les personnes et les actes visés par le recours}

Sauf s'ils sont protégés par la notion d'intérêt général, presque tous les propriétaires d'un fonds où se trouve de l'eau, qu'elle soit courante ou dormante, pourraient être exposés au recours. Même l'article 980 C.c.Q., qui semble accorder un droit au propriétaire d'un lac ou d'un étang entièrement situés sur son fonds, lui confère en réalité une obligation bien spécifique : il est tenu de conserver la qualité de l'eau de ce lac ou de cet étang. La loi ne lui accorde qu'une faculté donnée à tous, celle d'user de l'eau pour ses besoins, ce qui n'a pas pour effet de le soustraire au recours de l'article 982.

Le cas du propriétaire d'une source est différent : il peut en user et en disposer à sa guise, en autant qu'elle ne constitue pas la tête d'un cours d'eau (981 a contrario). Il semblerait qu'il échappe à la surveillance de 982 C.c.Q. Mais si la source constitue la tête d'un cours d'eau, il restera entièrement exposé au recours, d'autant plus que l'article 981 précise qu'il est tenu de conserver la qualité et la quantité de l'eau.

Peu de petits propriétaires, en fin de compte, échapperont à la surveillance de l'article 982. Ils ne pourront rien faire qui soit susceptible d'épuiser ou de polluer l'eau. En effet, l'emploi du verbe «éviter» pourrait, comme nous l'avons mentionné, suggérer que les ouvrages dont la modification ou la destruction peut être demandée n'ont pas à effectivement polluer ou épuiser l'eau : il suffirait qu'ils soient susceptibles de le faire éventuellement.

\section{Conclusion}

On peut donc reprocher à l'article 982 C.c.Q. d'ouvrir la porte à une interprétation qui soumettrait le droit de propriété à un vague droit d'usage, qui créerait un pouvoir de surveillance de tous sur chacun, et qui consacrerait, du même coup, l'interdiction de polluer et le droit de le faire.

En même temps que nous déplorons ce rôle qui leur incombe, nous pouvons souhaiter que les tribunaux circonscrivent ce recours nouveau, en fixent les critères, et lui imposent le cadre que le Législateur a omis de lui donner. Ainsi, il serait souhaitable qu'un intérêt distinct soit exigé pour sa mise en 
oeuvre $^{13}$ : la sanction qu'il crée ne devrait pouvoir être obtenue, tout au plus, que par celui qui est en mesure d'utiliser, et utilise effectivement l'eau dont il veut préserver la qualité ou la quantité. Ainsi, les recours de principe seraient évités, et le droit de propriété conserverait une apparence de cette complétude que le nouveau code lui prête encore ${ }^{14}$.

Nous pouvons également souhaiter que l'intérêt général empêchant l'exercice du recours reçoive une interprétation stricte, sinon restrictive, de façon à au moins soumettre les grandes sociétés polluantes au même régime que les petits propriétaires riverains.

13. Ce qui poserait un autre problème, comme nous l'avons déjà mentionné, en vidant de tout contenu l'obligation créée par l'article 980 al. 2 C.c.Q.

14. $\quad$ Article 947 C.c.Q. 